\title{
Prevalência de Estenose da Artéria Subclávia em Pacientes Candidatos a Cirurgia de Revascularização do Miocárdio: Registro Multicêntrico
}

\author{
Vitor Osório Gomes ${ }^{1}$, Marcelo Roman ${ }^{1}$, Christiano da Silveira de Barcellos ${ }^{1}$, Ricardo Lasevitch ${ }^{1}$, \\ Patrícia Hickmann ${ }^{1}$, Rafael Alcalde ${ }^{1}$, Alberto Brizolara', Norberto Duda², Rogério Tumelero², \\ Jorge A. Guimarães ${ }^{3}$, José Carlos Brito ${ }^{4}$, Itamar Oliveiras ${ }^{5}$, Maria Sanali Paiva ${ }^{5}$, Heitor Carvalho ${ }^{4}$, \\ Denise Carvalho ${ }^{1}$, Paulo Caramori ${ }^{1}$
}

\section{RESUMO}

Introdução: Aproximadamente $90 \%$ dos pacientes submetidos a cirurgia de revascularização do miocárdio (CRM) recebem enxerto de artéria torácica interna esquerda. A ocorrência de estenose da artéria subclávia esquerda pode resultar em falência do enxerto por limitação de fluxo coronário. A prevalência de estenose da artéria subclávia esquerda em pacientes com aterosclerose coronária grave, considerados candidatos a CRM, não é conhecida. Objetivo: Determinar a prevalência e a eficácia da avaliação clínica para diagnosticar a estenose da artéria subclávia esquerda em candidatos a CRM. Método: Em um registro multicêntrico, realizouse angiografia seletiva da artéria subclávia esquerda em pacientes com indicação de CRM na ocasião da cinecoronariografia. Estenoses maiores ou iguais a $50 \%$ antes da origem da artéria torácica interna foram consideradas significativas. Resultados: Dos 205 pacientes estudados, 16 (7,8\%) apresentavam estenose significativa da artéria subclávia esquerda. A medida da pressão arterial não-invasiva diferencial entre os membros superiores $\geq 10 \mathrm{mmHg}$ teve baixa sensibilidade $(37,5 \%)$ e baixo valor preditivo positivo $(13,3 \%)$ para a identificação da estenose. Não foram identificados preditores clínicos de estenose da artéria subclávia pela análise univariada. Conclusão: Estenose da artéria subclávia esquerda não é incomum em pacientes com indicação de CRM. O gradiente de pressão entre os membros superiores é um método diagnóstico insuficiente. Portanto, em pacientes candidatos a CRM, a realização de arteriografia seletiva da artéria subclávia esquerda deve ser considerada, pelo risco de comprometimento de fluxo e síndrome do roubo coronário-subclávio em pacientes com estenose da subclávia não diagnosticada que recebem enxerto de artéria torácica interna esquerda.

DESCRITORES: Aterosclerose. Artéria subclávia/anormalidades. Síndrome do roubo subclávio. Angiografia.

1 Serviço de Hemodinâmica - Hospital São Lucas-PUCRS - Porto Alegre, RS.

${ }^{2}$ Hospital São Vicente de Paulo - Passo Fundo, RS.

3 Hospital Português - Recife, PE.

${ }^{4}$ Hospital Santa Izabel - Salvador, BA.

5 InCor - Natal, RN.

Correspondência: Paulo Caramori. Serviço de Hemodinâmica - Hospital São Lucas-PUCRS. Av. Ipiranga, 6.690 - Jardim Botânico - Porto Alegre, RS - CEP 90619-900 - E-mail: intervcard.@pucrs.br

Recebido em: 5/5/2008 • Aceito em: 24/8/2008

\section{SUMMARY}

Prevalence of Stenosis of the Left Subclavian Artery in CABG Candidates: A Multicenter Registry

Background: Approximately $90 \%$ of the patients submitted to coronary artery bypass graft surgery (CABG) receive a left internal thoracic artery (LITA) graft. Stenosis of the left subclavian artery can result in graft failure due to restricted coronary flow. The prevalence of stenosis of the left subclavian artery in patients with severe coronary atherosclerosis, deemed candidates to CABG, is not known. Objective: To assess the prevalence of stenosis of left subclavian artery in CABG candidates, as well as the diagnostic effectiveness of clinical evaluation. Methods: In a multicenter registry, selective angiography of the left subclavian artery was carried out in patients considered candidates to CABG during coronary cineangiography. Stenoses $\geq 50 \%$ proximal to the origin of the LITA were considered significant. Results: A total of 205 patients were included. Significant stenosis of the left subclavian artery was observed in $16(7.8 \%)$ patients. Noninvasive differential arterial blood pressure measurement $\geq 10 \mathrm{mmHg}$ between both arms showed low sensitivity (37.5\%) and low positive predictive value $(13.3 \%)$ for stenosis identification. No clinical predictors of significant stenosis were identified by univariate analysis. Conclusion: Stenosis of the left subclavian artery is not uncommon in CABG candidates. Noninvasive blood pressure gradient between both arms has low diagnostic accuracy. Therefore, in patients candidates to CABG, selective angiography of the left subclavian artery should be considered, because of the risk of reduced flow and coronary-subclavian steal syndrome in patients with non-diagnosed stenosis of the subclavian artery who receive a LITA graft.

DESCRIPTORS: Atherosclerosis. Subclavian artery/abnormalities. Subclavian steal syndrome. Angiography. 
O S avanços da cardiologia intervencionista têm feito da angioplastia coronária com stents intracoronários uma importante opção terapêutica para pacientes com doença arterial coronária. Dessa forma, atualmente, os pacientes encaminhados para cirurgia de revascularização do miocárdio (CRM) tendem a ter doença coronária mais extensa, freqüentemente associada a aterosclerose em outros territórios vasculares.

Aproximadamente $90 \%$ dos pacientes submetidos a CRM recebem enxerto de artéria torácica interna esquerda, pois esse enxerto proporciona ao paciente maior sobrevida em comparação aos que recebem apenas enxerto de veia safena ${ }^{1}$. Alguns estudos indicam que em uma proporção de pacientes que receberam enxerto de artéria torácica interna esquerda há estenose da artéria subclávia esquerda à jusante da mesma. Essa estenose pode potencialmente resultar em síndrome do roubo de fluxo coronário-subclávio e déficit de fluxo coronário a partir do enxerto. Por esse motivo, o diagnóstico de estenose da artéria subclávia pode ter implicações terapêuticas pela necessidade de correção cirúrgica ou percutânea ${ }^{2,3}$, antes mesmo da CRM ${ }^{4,5}$.

A prevalência de estenose da artéria subclávia esquerda em pacientes candidatos a CRM em séries internacionais varia de $1 \%$ a $14 \%$, conforme o risco da população estudada ${ }^{6-8}$. Existem poucos dados na literatura, especialmente estudos multicêntricos, avaliando a prevalência desse problema, bem como critérios que determinem quais pacientes devam ser estudados sistematicamente.

O objetivo deste estudo foi descrever a prevalência de estenose da artéria subclávia esquerda em pacientes candidatos a CRM submetidos a cateterismo cardíaco, bem como determinar a capacidade de diagnosticar essa estenose pela avaliação clínica.

\section{MÉTODO}

\section{Delineamento do estudo}

Esse é um estudo transversal realizado a partir de um registro multicêntrico que avaliou a prevalência de estenose da artéria subclávia esquerda em pacientes candidatos a CRM. No período de janeiro de 2004 a julho de 2007, foram incluídos consecutivamente no registro pacientes de ambos os sexos, com mais de 18 anos. O critério de seleção dos pacientes baseou-se na cineangiocoronariografia. Foram incluídos pacientes considerados candidatos a CRM pelo hemodinamicista, com base na gravidade da doença arterial coronária.

Foram excluídos pacientes com contra-indicação clínica para CRM ou que tenham se recusado a participar do estudo. Todos os pacientes submetidos a cineangiocoronariografia no período de realização do estudo assinaram o termo de consentimento, considerando a necessidade de realização de angiografia adicional. O trabalho foi aprovado pelos comitês científico e de ética do centro coordenador.
O estudo foi desenvolvido no Serviço de Hemodinâmica do Hospital São Lucas da Pontifícia Universidade Católica do Rio Grande do Sul (centro coordenador), em Porto Alegre, RS, no Hospital InCor de Natal, RN, no Hospital Santa Izabel, em Salvador, BA, no Hospital São Vicente de Paulo, em Passo Fundo, RS, e no Hospital Real Português, no Recife, PE.

\section{Coleta dos dados}

As características demográficas, antropométricas e clínicas dos pacientes, as medidas de pressão arterial dos membros superiores e as angiografias foram registradas de forma padronizada antes da realização da cineangiocoronariografia. O centro coordenador foi responsável por tabular os dados e realizar angiografia quantitativa off line. Não houve a medida do gradiente translesional, tendo sido objeto deste estudo o gradiente entre os membros superiores medido por esfigmomanometria.

\section{Angiografia}

Nos pacientes com indicação de CRM, a angiografia seletiva da artéria subclávia esquerda foi realizada imediatamente após a cinecoronariografia, com cateter JR 4, e $10 \mathrm{ml}$ de contraste na projeção ântero-posterior. Incidências adicionais foram utilizadas a critério do examinador. A incidência que melhor identificasse a artéria subclávia esquerda foi analisada quantitativamente por dois hemodinamicistas, utilizando-se o software Image ${ }^{\circledR}$ (National Institute of Health, Estados Unidos). Foram consideradas estenoses significativas aquelas $\geq 50 \%$.

\section{Análise estatística}

O tamanho da amostra foi calculado por método de comparação de proporções com prevalência estimada (a partir da literatura), na população de pacientes candidatos a $\mathrm{CRM}^{9}$, de $5 \%$ e precisão da estimativa de $3 \%$ para mais ou para menos, o que resultou em amostragem calculada de 205 pacientes.

O teste $t$ de Student e o teste de Mann-Whitney foram utilizados para comparar as variáveis contínuas entre pacientes com e sem estenose da artéria subclávia. As variáveis qualitativas foram analisadas pelos testes do qui-quadrado e exato de Fischer. Foi realizada análise descritiva das características da população, bem como da prevalência de estenose da artéria subclávia esquerda. Os fatores de risco para estenose significativa foram avaliados por análise univariada. As diferenças foram consideradas significativas para valores de $p \leq 0,05$.

\section{RESULTADOS}

Um total de 205 pacientes foi estudado, com média de idade de 63,2 anos, sendo 135 (65,9\%) homens. Estenoses maiores que $50 \%$ da artéria subclávia esquerda foram identificadas em $16(7,8 \%)$ pacientes, com dois casos de oclusão total do vaso (Figura 1). Na 
Gomes VO, et al. Prevalência de Estenose da Artéria Subclávia em Pacientes Candidatos a Cirurgia de Revascularização do Miocárdio: Registro Multicêntrico. Rev Bras Cardiol Invas. 2008;16(3):307-311.

análise univariada, nenhuma característica clínica, demográfica ou angiográfica se mostrou fator preditor para ocorrência de estenose subclávia (Tabela 1). Nenhum paciente apresentava sinais ou sintomas de atrofia ou isquemia do membro superior esquerdo ou de insuficiência vertebrobasilar.

O gradiente de pressão arterial não-invasiva entre membro superior direito e membro superior esquerdo medido por esfigmomanômetro maior que $10 \mathrm{mmHg}$ teve baixa sensibilidade $(37,5 \%)$ e baixo valor preditivo positivo $(13,3 \%)$ para a identificação da estenose (Tabela 2).

\section{DISCUSSÃO}

Os resultados deste estudo demonstram que, em nosso meio, uma taxa considerável de pacientes candidatos a CRM apresenta estenose da artéria subclávia esquerda não diagnosticada.

Na literatura, a prevalência de estenose da artéria subclávia esquerda em uma população não-selecionada de indivíduos submetidos a angiografia coronária varia de $0,5 \%$ a $6,8 \%{ }^{10-13}$. Em pacientes com evidências clínicas de doença arterial periférica, essa prevalência varia de $11,8 \%$ a $18,7 \%{ }^{14}$

Outro achado interessante do presente estudo é que na população de pacientes candidatos a CRM a

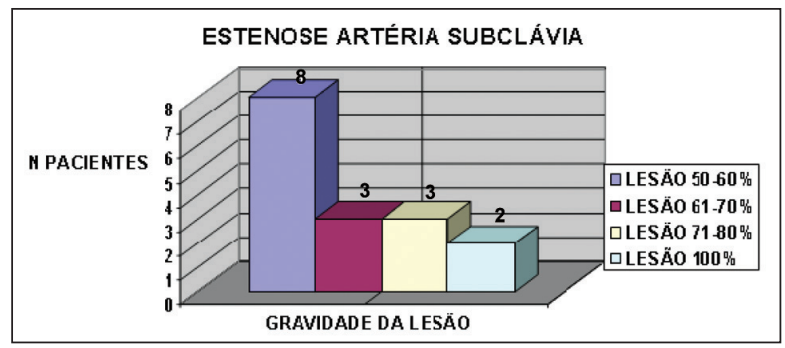

Figura 1 - Pacientes com estenose significativa da artéria subclávia. medida do gradiente de pressão entre os membros superiores não foi suficientemente acurada para predizer a ocorrência de estenose da artéria subclávia esquerda na angiografia, com valor preditivo positivo de apenas $13,3 \%$ para gradiente maior que $10 \mathrm{mmHg}$.

A utilização do gradiente sistólico entre os membros superiores, como método diagnóstico, tem resultados conflitantes em publicações prévias ${ }^{12,13,15,16}$ (Tabela 3).

Em uma coorte avaliando 2.975 indivíduos saudáveis, como grupo de controle, comparados a 1.248 pacientes com fatores de risco cardiovascular, a prevalência de estenose da artéria subclávia, definida como gradiente de pressão sistólica entre os membros superiores maior que $15 \mathrm{mmHg}$, foi de 1,9\% e 7,1\%, respectivamente. Nessa coorte, a história de doença arterial periférica foi forte preditor de estenose da artéria subclávia ${ }^{17}$. Em nosso estudo, a análise univariada não identificou fatores de risco para estenose angiográfica da artéria subclávia esquerda.

Outros achados clínicos, como história de acidente vascular cerebral, sopros cervicais e/ou supraclaviculares, claudicação do membro superior esquerdo ou assimetria de pulsos, embora sugestivos, habitualmente apresentam acurácia diagnóstica ainda menor ${ }^{18}$.

Deve-se ressaltar que este registro avalia a prevalência de estenose da artéria subclávia numa população de interesse real, quais sejam, pacientes candidatos a CRM nos quais há impacto potencial na identificação de estenose assintomática da artéria subclávia esquerda. Essa informação pode auxiliar a estabelecer novas indicações de arteriografia seletiva da artéria subclávia, uma vez que, na maioria dos serviços, somente é realizada na ocorrência de sintomas ou sinais de hipoperfusão do membro superior ou insuficiência vertebrobasilar.

A partir do diagnóstico de estenose da artéria subclávia esquerda, as opções terapêuticas dependem do

TABELA 1

Características demográficas e clínicas da população e incidência de estenose angiográfica de subclávia esquerda

\begin{tabular}{lccc}
\hline & Sem estenose $(\mathbf{n}=\mathbf{1 8 9})$ & Estenose $\mathbf{2} \mathbf{5 0} \mathbf{( n = 1 6 )}$ & $\mathbf{p}$ \\
\hline Idade, anos & $62 \pm 11$ & $69 \pm 7$ & 0,17 \\
Sexo masculino & $56 \%$ & $66 \%$ & 0,21 \\
Dislipidemia & $69 \%$ & $68 \%$ & $>0,99$ \\
Hipertensão arterial & $77 \%$ & $87 \%$ & 0,53 \\
Diabetes melito & $29 \%$ & $31 \%$ & 0,32 \\
Tabagismo & $45 \%$ & $50 \%$ & 0,47 \\
Doença vascular periférica & $22 \%$ & $31 \%$ & 0,37 \\
Acidente vascular cerebral prévio & $9 \%$ & $13 \%$ & 0,65 \\
\hline
\end{tabular}

$\mathrm{n}=$ número de pacientes. 
TABELA 2

Desempenho diagnóstico do diferencial da pressão arterial nos membros superiores com esfigmomanômetro

\begin{tabular}{|c|c|c|c|c|}
\hline & Sensibilidade & Especificidade & VPP & VPN \\
\hline$\Delta \mathrm{PAS}>10 \mathrm{mmHg}$ & $37,5 \%$ & $79,8 \%$ & $13,3 \%$ & $93,7 \%$ \\
\hline
\end{tabular}

TABELA 3

Valor preditivo da pressão arterial entre membro superior direito e membro superior esquerdo para diagnóstico de estenose da artéria subclávia na literatura

\begin{tabular}{lcccc}
\hline Autor & Pacientes & $\boldsymbol{\Delta}$ PA MSD-MSE (mmHg) & VPP (\%) & VPN (\%) \\
\hline Osborn et al. $^{12}$ & 59 & $\geq 15$ & 100 & 100 \\
Lobato et al. $^{15}$ & 86 & $\geq 20$ & 67 & 100 \\
Mulvihill et al. $^{16}$ & 6 & $>10$ & 50 & $\mathrm{n} / \mathrm{a}$ \\
English et al. $^{8}$ & 492 & $>10$ & 13 & 99 \\
English et al. & 492 & $\geq 20$ & 19 & 98 \\
\hline
\end{tabular}

$\triangle$ PA MSD-MSE $(\mathrm{mmHg})=$ gradiente da pressão arterial sistólica entre o membro superior direito e o membro superior esquerdo em milímetros de mercúrio; $\mathrm{n} / \mathrm{a}=$ não avaliado; $\mathrm{VPN}=$ valor preditivo negativo; $\mathrm{VPP}=$ valor preditivo positivo.

quadro clínico de apresentação do paciente. O bypass cirúrgico aorto-axilar ou carotídeo-subclávio é uma opção, com resultados de patência superior a 90\% em seguimento médio de 6,3 anos, com baixa morbidade e mortalidade ${ }^{19,20}$. A estratégia percutânea foi comparada a resultados cirúrgicos, demonstrando igual efetividade e menor índice de complicações, sendo considerada a primeira opção de tratamento nas obstruções da artéria subclávia e do tronco braquiocefálico ${ }^{20}$. A intervenção percutânea antes da CRM tem sido o método de eleição naqueles pacientes que não necessitam cirurgia de emergência. A ocorrência de reestenose em várias séries é menor que $10 \%$ e a ocorrência de trombose é rara ${ }^{21-23}$. A conduta terapêutica em pacientes que necessitam de CRM em caráter de urgência deve considerar a utilização da artéria torácica interna direita (artéria mamária direita) ou da artéria torácica interna esquerda (artéria mamária esquerda) como enxerto livre, o que parece assegurar índices de patência comparáveis aos do enxerto in situ para a artéria descendente anterior ${ }^{24-26}$.

O desenvolvimento de síndrome do roubo coronáriosubclávio não pode ser predito no pré ou no pósoperatório imediato, podendo ocorrer dois a trinta anos após a $\mathrm{CRM}^{27}$. Sua ocorrência deve ser suspeitada em pacientes com angina recorrente após CRM com uso de artéria torácica interna esquerda cujo gradiente de pressão entre os membros superiores seja evidente ${ }^{28}$. A prevalência é muito variada na literatura, na dependência do tempo de seguimento, podendo ser tão incomum quanto $0,07 \%$ ou tão prevalente quanto $3,4 \%$ dos pacientes submetidos a $\mathrm{CRM}^{15}$.

Finalmente, nos pacientes que apresentam síndrome do roubo coronário-subclávio, o tratamento percutâneo é a primeira opção e sua eficácia tem sido demonstrada em vários estudos ${ }^{22,27}$.

A principal limitação do presente estudo é a pequena amostra de pacientes incluídos. Outra limitação está relacionada ao fato de a medida do gradiente translesional não ter sido realizada, impossibilitando avaliar o real impacto funcional da lesão da artéria subclávia detectada angiograficamente.

\section{CONCLUSÃO}

Neste registro multicêntrico de pacientes candidatos a CRM, a prevalência de estenose da artéria subclávia esquerda foi de $7,8 \%$. Os sintomas e sinais clínicos demonstraram sensibilidade e valor preditivo baixos para o diagnóstico de estenose significativa da artéria subclávia esquerda proximal à origem da artéria mamária esquerda. As implicações clínicas deste achado podem ser relevantes na população de pacientes candidatos a CRM.

Dessa forma, a realização de angiografia seletiva da artéria subclávia esquerda talvez deva ser considerada no momento da cinecoronariografia ao se identificar pacientes candidatos a CRM, uma vez que a prevalência de estenose da subclávia não é desprezível nessa população. 
Gomes VO, et al. Prevalência de Estenose da Artéria Subclávia em Pacientes Candidatos a Cirurgia de Revascularização do Miocárdio: Registro Multicêntrico. Rev Bras Cardiol Invas. 2008;16(3):307-311.

\section{REFERÊNCIAS BIBLIOGRÁFICAS}

1. Loop FD, Lytle BW, Cosgrove DM, Stewart RW, Goormastic $M$, Williams GW, et al. Influence of internal-mammaryartery graft on 10-year survival and other cardiac events. $\mathrm{N}$ Engl J Med. 1986;314(1):1-6.

2. Bachman DM, Kim RM. Transluminal dilatation for subclavian steal syndrome. AJR Am J Roentgenol. 1980;135(5):995-6.

3. Kumar K, Dorros G, Bates MC, Palmer L, Mathiak L, Dufek C. Primary stent deployment in occlusive subclavian artery disease. Cathet Cardiovasc Diagn. 1995;34(4):281-5.

4. Kugelmass AD, Kim D, Kuntz RE, Carrozza JP Jr, Baim DS. Endoluminal stenting of a subclavian artery stenosis to treat ischemia in the distribution of a patent left internal mammary graft. Cathet Cardiovasc Diagn. 1994;33(2):175-7.

5. Perrault LP, Carrier M, Hudon G, Lemarbre L, Hébert $Y$, Pelletier LC. Transluminal angioplasty of the subclavian artery in patients with internal mammary grafts. Ann Thorac Surg. 1993;56(4):927-30.

6. Krijne R, Deng MC, Heinrich KW, Sons H, Krian A. Semiselective angiography of the internal mammary arteries as preparation for coronary bypass surgery. Am J Cardiol. 1990;66(3):377-8.

7. Singh RN. Atherosclerosis and the internal mammary arteries. Cardiovasc Intervent Radiol. 1983;6(2):72-7.

8. English JA, Carell ES, Guidera SA, Tripp HF. Angiographic prevalence and clinical predictors of the left subclavian stenosis in patients undergoing diagnostic cardiac catheterization. Catheter Cardiovasc Interv. 2001;54(1):8-11.

9. Lwanga SK, Lemeshow S. Sample size determination in health studies: a practical manual. Geneva: World Health Organization;1991.

10. Olsen CO, Dunton RF, Maggs PR, Lahey SJ. Review of coronary-subclavian steal following internal mammary artery-coronary artery bypass surgery. Ann Thorac Surg. 1988;46 (6):675-8

11. Feit A, Reddy CV, Cowley C, Ibrahim B, Zisbrod Z. Internal mammary artery angiography should be a routine component of diagnostic coronary angiography. Cathet Cardiovasc Diagn. 1992;25(2):85-90.

12. Osborn LA, Vernon SM, Reynolds B, Timm TC, Allen K. Screening for subclavian artery stenosis in patients who are candidates for coronary bypass surgery. Catheter Cardiovasc Interv. 2002;56(2):162-5.

13. English JA, Carell ES, Guidera SA, Tripp HF. Angiographic prevalence and clinical predictors of left subclavian stenosis in patients undergoing diagnostic cardiac catheterization. Catheter Cardiovasc Interv. 2001;54(1):8-11.

14. Gutierrez GR, Mahrer $P$, Aharonian V, Mansukhani $P$, Bruss J. Prevalence of subclavian artery stenosis in patients with peripheral vascular disease. Angiology. 2001;52(3):189-94.

15. Lobato EB, Kern KB, Bauder-Heit J, Hughes L, Sulek CA.
Incidence of coronary-subclavian steal syndrome in patients undergoing noncardiac surgery. J Cardiothorac Vasc Anesth. 2001;15(6):689-92.

16. Mulvihill NT, Loutfi M, Salengro E, Boccalatte M, Laborde JC, Fajadet J, et al. Percutaneous treatment of coronary subclavian steal syndrome. J Invasive Cardiol. 2003;15(7):390-2.

17. Shadman R, Criqui MH, Bundens WP, Fronek A, Denenberg JO, Gamst AC, et al. Subclavian artery stenosis: prevalence, risk factors, and association with cardiovascular diseases. J Am Coll Cardiol. 2004;44(3):618-23.

18. Rogers JH, Calhoun RF $2^{\text {nd }}$. Diagnosis and management of subclavian artery stenosis prior to coronary artery bypass grafting in the current era. J Card Surg. 2007;22(1):20-5.

19. Ochi M, Hatori N, Hinokiyama K, Saji Y, Tanaka S. Subclavian artery reconstruction in patients undergoing coronary artery bypass grafting. Ann Thorac Cardiovasc Surg. $2003 ; 9(1): 57-61$.

20. Hadjipetrou P, Cox S, Piemonte T, Eisenhauer A. Percutaneous revascularization of atherosclerotic obstruction of aortic arch vessels. J Am Coll Cardiol. 1999;33(5):1238-45.

21. Henry M, Amor M, Henry I, Ethevenot G, Tzvetanov K, Chati Z. Percutaneous transluminal angioplasty of the subclavian arteries. J Endovasc Surg. 1999;6(1):33-41.

22. Millaire A, Trinca M, Marache $P$, de Groote $P$, Jabinet JL, Ducloux G. Subclavian angioplasty: Immediate and late results in 50 patients. Cathet Cardiovasc Diagn. 1993;29(1):8-17.

23. Mufti SI, Young KR, Schulthesis T. Restenosis following subclavian artery angioplasty for treatment of coronary-subclavian steal syndrome: definitive treatment with Palmaz-stent placement. Cathet Cardiovasc Diagn. 1994;33(2):172-4.

24. Tatoulis J, Buxton BF, Fuller JA. Patencies of 2127 arterial to coronary conduits over 15 years. Ann Thorac Surg. 2004;77 (1):93-101 .

25. Tashiro T, Nakamura K, Sukehiro S, Nakamura M, Shibano $\mathrm{R}$, Motomura $\mathrm{T}$, et al. Midterm results of free internal thoracic artery grafting for myocardial revascularization. Ann Thorac Surg. 1998;65(4):951-4.

26. Yamashiro S, Sakata R, Nakayama Y, Ura M, Arai Y, Morishima $Y$. Long-term angiographic evaluation of free internal thoracic artery grafting for myocardial revascularization. Ann Thorac Cardiovasc Surg. 2000;6(6):378-82.

27. Westerband A, Rodriguez JA, Ramaiah VG, Diethrich EB. Endovascular therapy in prevention and management of coronary-subclavian steal. J Vasc Surg. 2003;38(4):699-703.

28. Celkan MA, Daglar B, Kazaz H, Dinckal H. Symptomatic coronary-subclavian steal syndrome due to total occlusion of proximal left subclavian artery. Interact Cardiovasc Thorac Surg. $2003 ; 2(4): 534-6$.

29. Takach TJ, Reul GJ, Gregoric I, Krajcer Z, Duncan JM, Livesay J, et al. Concomitant subclavian and coronary artery disease. Ann Thorac Surg. 2001;71(1):187-9. 